\title{
Overt and covert attention shifts to emotional faces - combining EEG, Eye-tracking and a Go/No-go paradigm
}

\author{
Louisa Kulke $e^{1,2,3^{*}}$, Lena Brümmer ${ }^{1}$, Arezoo Pooresmaeilii, ${ }^{3,4}$ Annekathrin Schacht ${ }^{1,3}$ \\ 1) Georg-August-University Göttingen, ${ }^{2)}$ Friedrich-Alexander Universität Erlangen-Nürnberg, \\ ${ }^{3)}$ Leibniz-ScienceCampus Primate Cognition, ${ }^{4)}$ European Neuroscience Institute Göttingen \\ *Corresponding authorLouisa Kulke: louisa.kulke@fau.de
}

In everyday life, faces with emotional expressions quickly attract attention and eye-movements. To study the neural mechanisms of such emotion-driven attention by means of event-related brain potentials (ERPs), tasks that employ covert shifts of attention are commonly used, in which participants need to inhibit natural eye-movements towards stimuli. It remains, however, unclear how shifts of attention to emotional faces with and without eye-movements differ from each other. The current preregistered study aimed to investigate neural differences between covert and overt emotion-driven attention. We combined eye-tracking with measurements of ERPs to compare shifts of attention to faces with happy, angry or neutral expressions when eyemovements were either executed (Go conditions) or withheld (No-go conditions). Happy and angry faces led to larger EPN amplitudes, shorter latencies of the P1 component and faster saccades, suggesting that emotional expressions significantly affected shifts of attention. Several ERPs (N170, EPN, LPC), were augmented in amplitude when attention was shifted with an eye-movement, indicating an enhanced neural processing of faces if eye-movements had to be executed together with a reallocation of attention. However, the modulation of ERPs by facial expressions did not differ between the Go and No-go conditions, suggesting that emotional content enhances both covert and overt shifts of attention. In summary, our results indicate that overt and covert attention shifts differ but are comparably affected by emotional content.

Key words: Overt attention; Covert attention; Emotion-driven attention; ERPs; Eye-movements

\section{Introduction}

Emotional faces quickly attract our attention (Junghöfer et al., 2006; Pourtois, Grandjean, Sander, \& Vuilleumier, 2004) and gaze (Hunt, Cooper, Hungr, \& Kingstone, 2007), even when presented below the threshold of awareness (Vetter, Badde, Phelps, \& Carrasco, 2019). However, some social situations warrant that direct eye-movements towards other people (i.e. overt shifts of attention) are inhibited avoiding awkward situations when incidentally looking at strangers (Foulsham, Walker, \& Kingstone, 2011; Hayward, Voorhies, Morris, Capozzi, \& Ristic, 2017; Laidlaw, Foulsham, Kuhn, \&
Kingstone, 2011). Behavioral studies have shown that people prefer to look at faces over other stimuli on a computer screen but avoid looking at them in everyday settings (Foulsham et al., 2011; Hayward et al., 2017; Laidlaw et al., 2011). Even if no eyemovements take place, attention may still be shifted towards other people's faces without looking at them (covert attention shift). Consequently, to get a clear picture of attention in real life situations, both overt and covert attention shifts need to be considered. However, the neural mechanisms of overt and covert attention shifts to emotional faces, where attentional shifts are accompanied by an overt shift of 
gaze or not, have never been directly compared in a paradigm that manipulates both types of attention shift. Such a comparison is however crucial, to relate findings from the new stream of real-life studies involving both overt and covert attention (Argyle \& Cook, 1976; Gobel, Kim, \& Richardson, 2015; Nasiopoulos, Risko, \& Kingstone, 2015; Risko, Richardson, \& Kingstone, 2016) to laboratory-based Electroencephalography (EEG) tasks that often require only covert attention. The central aim of this study is to undertake this comparison and directly test the neural mechanisms of overt and covert shifts of attention to emotional stimuli against each other.

There is ample evidence that emotional content increases the overall perceptual salience of faces. For instance, it has been shown that enhanced salience of emotional (e.g. fearful) faces results in a facilitation of their detection compared to neutral faces (Bayle, Schoendorff, Hénaff, \& KrolakSalmon, 2011) and leads to a higher propensity of eye-movements towards them (Bannerman, Hibbard, Chalmers, \& Sahraie, 2012; Kissler \& Keil, 2008; Nummenmaa, Hyönä, \& Calvo, 2006). However, when fast, reflexive first saccades to face stimuli were investigated, no effect of expression was found (Kulke, 2019).Together these studies suggest that emotional salience and physical salience may have similar behavioral and neural correlates. Whereas the neural correlates of covert and overt shifts of attention to emotionally salient stimuli have not been tested before, previous research has examined the underlying neural mechanisms of the two types of attention shifts. For instance, Kulke, Atkinson, and Braddick (2016a) combined eye-tracking and EEG with a fixation shift paradigm (Atkinson, Hood, Braddick, \& WattamBell, 1988; Atkinson, Hood, Wattam-Bell, \& Braddick, 1992; Hood \& Atkinson, 1993; Kulke, Atkinson, \& Braddick, 2015) and asked participants to either make an overt attention shift (i.e. involving an eyemovement) towards a peripheral target, or to make a covert attention shift (i.e. keep fixating their gaze on the center). The study used high contrast bars as target stimuli, demonstrating that mechanisms for covert and overt attention shifts to such physically salient targets are similar in occipital areas (Kulke et al., 2016a), but subtly differ in frontal responses, possibly due to the inhibition of eye-movements (Bokura, Yamaguchi, \& Kobayashi, 2001; Kulke et al., 2016a). However, it is unknown whether these findings also extend to situations where socio-emotional relevance rather than physical salience is enhanced.

Neural responses to faces differ from those to simple physically salient targets. Faces are selectively processed in the fusiform face area (Kanwisher, McDermott, \& Chun, 1997), where neurons are more responsive to faces than other objects, among others, and lead to enhanced neural responses, in particular of the N170 (Bentin, Allison, Puce, Perez, \& McCarthy, 1996; Eimer, 2000; Itier \& Taylor, 2004). Previous studies reported larger Event-Related brain Potential (ERP) responses towards emotional compared to neutral faces at different levels of processing, including the P1, N170, Early Posterior Negativity (EPN) and Late Positive Component (LPC) (Batty \& Taylor, 2003; Hinojosa, Méndez-Bértolo, \& Pozo, 2010; Hinojosa, Mercado, \& Carretié, 2015; Rellecke, Sommer, \& Schacht, 2012; Schacht \& Sommer, 2009; Schupp, Junghöfer, Weike, \& Hamm, 2004), although the earliest time when emotion effects start to emerge remains unclear (for a recent review, see Schindler \& Bublatzky, 2020). While some authors found very early neural differences between neutral and emotional faces on the $\mathrm{C} 1$ (Pourtois et al., 2004), P1 (Hammerschmidt, Sennhenn-Reulen, \& Schacht, 2017; Pourtois, Dan, Grandjean, Sander, \& Vuilleumier, 2005; Rellecke, Palazova, Sommer, \& Schacht, 2011; Vlamings, Goffaux, \& Kemner, 2009) or N170 (for reviews, see Hinojosa et al., 2015; Rellecke, Sommer, \& Schacht, 2013), other studies failed to find such early emotion effects on the P1 (Frühholz, 
Jellinghaus, \& Herrmann, 2011; Rossignol, Philippot, Bissot, Rigoulot, \& Campanella, 2012) or N170 (Eimer \& Holmes, 2002). The onset of emotion effects on ERPs therefore remains unclear and has mainly been studied in lab-based tasks, where participants are instructed to inhibit eyemovements (for a recent review, see Schindler \& Bublatzky, 2020).

An important reason that electrophysiological underpinnings of overt emotion-driven attention are not well characterized is that eye-movements typically lead to artefacts in EEG data (Anllo-Vento \& Hillyard, 1996; Eimer, Forster, Velzen, \& Prabhu, 2005; Eimer, Van Velzen, \& Driver, 2002; Hopfinger \& Mangun, 1998; Martinez et al., 1999; Praamstra \& Oostenveld, 2003; Shomstein, Kravitz, \& Behrmann, 2012; Yamaguchi, Tsuchiya, \& Kobayashi, 1994, 1995). To accommodate for this, most previous studies have resorted to measuring covert shifts of attention. More recently it became possible to investigate overt attention shifts by combining EEG and eye-tracking (Dimigen, Sommer, Hohlfeld, Jacobs, \& Kliegl, 2011; Huber-Huber, Ditye, Fernández, \& Ansorge, 2016; Kulke, 2015, 2019; Kulke et al., 2016a; Kulke, Atkinson, \& Braddick, 2016b, 2020; Weaver, van Zoest, \& Hickey, 2017). Specifically, two studies (Kulke et al., 2016a, 2020) combined eye-tracking and EEG to investigate the neural mechanisms of covert and overt attention shifts to physical salience. These previous studies thus suggest that eye-tracking and EEG recording can be successfully combined to investigate neural correlates of overt attentional shifts, despite eye-movement related artefacts in EEG data.

While previous ERP research mainly focused on covert attention, fMRI studies have undertaken a comparison of covert and overt attention to neutral stimuli (Phillips et al., 2004)(cite the paper in comment) and found either overlapping (Beauchamp, Petit, Ellmore, Ingeholm, \& Haxby, 2001; De Haan, Morgan, \& Rorden, 2008; A.
Nobre, D. Gitelman, E. Dias, \& M. Mesulam, 2000) or distinct neural correlates for the two types of attention (Fairhall, Indovina, Driver, \& Macaluso, 2009). Another related line of fMRI research compared overt and covert presentation of emotional faces that could elicit different degrees of awareness of emotional content (Phillips et al., 2004; Sabatini et al., 2009). However, these previous studies did not test emotion-driven attention shifts. Across various neuroimaging techniques, only one study investigated ERPs related to reflexive overt attention shifts to emotional faces (Kulke, 2019). The findings suggest that during overt attention shifts emotional expressions of the target faces impact only those ERPs that occur after the eyemovements have been completed (EPN) but not early responses (P1) before the saccades. To our knowledge, attention shifts with and without eye-movements to emotional faces have never been directly compared. It therefore remains unclear, whether brain mechanisms of attention shifts to emotional faces differ depending on whether eye-movements occur or not and whether those differences are modulated by emotional content that the faces convey. If eye-movements play a crucial role when humans gather emotional information about others, emotion effects should be significantly enhanced when they are accompanied by an eye-movement (i.e. during overt attention shifts). If, however, emotion can just as reliably be processed during covert attention shifts, no differences in emotion effects should occur when the eyes are moved towards the emotional object, suggesting that no direct gaze is required to evaluate other people's emotional state during social interactions.

\subsection{Aims and Hypotheses}

The present pre-registered (https://osf.io/4kscq) study aimed to investigate neural differences in shifts of attention with and without eye-movements, depending on whether the target is a neutral compared to an emotional (happy, angry) face. A Go/No-go task was used, in which 
participants were explicitly instructed to make an eye-movement towards peripheral faces (Go) or not (No-go condition). These explicit instruction conditions were used to manipulate overt and covert attention in a controlled fashion, although it should be noted that both types of attention shifts are freely selected during natural gaze. It has been suggested that attention is shifted covertly towards briefly presented targets even if eye-movements need to be suppressed (Belopolsky \& Theeuwes, 2012; Mulckhuyse, Talsma, \& Theeuwes, 2007; Van der Stigchel \& Theeuwes, 2007). In our coregistration set up, eye-tracking was used to measure whether and how quickly attention is shifted overtly, while simultaneous EEG was used to measure underlying neural mechanisms. We expected neural responses to be affected by the emotional expression of the target and by the overtness of an attention shift. Specifically, based on previous behavioral findings (Bannerman et al., 2012; Bayle et al., 2011; Kissler \& Keil, 2008; Nummenmaa et al., 2006), we predicted that the attentional draw of emotional stimuli makes it difficult to inhibit eye-movements towards them. Therefore, we expected shorter saccade and $\mathrm{P} 1$ latencies in response to emotional compared to neutral faces and more errors (i.e., erratic saccades in No-go trials with emotional compared to neutral facial expressions). As frontal regions have been found to respond to saccade inhibition effort (Bokura et al., 2001; Kulke et al., 2016a), we expected larger frontal responses in No-go compared to Go conditions, varying with emotional expression. We further expected enhanced amplitudes of later ERP components (N170, EPN and LPC) in response to emotional than to neutral faces, independent of saccade execution. As emotional processing differs between individuals (Hamann \& Canli, 2004; Kaltwasser, Hildebrandt, Recio, Wilhelm, \& Sommer, 2014; Recio, Wilhelm, Sommer, \& Hildebrandt, 2017), particularly depending on social anxiety (Bradley, Mogg, \& Millar, 2000; Mogg, Philippot, \& Bradley, 2004; Wieser,
Hambach, \& Weymar, 2018; Wieser, Pauli, Weyers, Alpers, \& Mühlberger, 2009), participants were preselected for this study to test a wide distribution of non-clinical differences in social anxiety and findings regarding individual differences are reported in Supplement A.

In summary, the current study aimed to investigate the effects of (a) emotion and (b) eye-movements on neural mechanisms of attention. It sought to disentangle the mechanisms involved in covert attention shifting paradigms, as used in the previous literature and overt shifts, which occur in everyday life, thereby expanding our knowledge of the neural mechanisms that underlie processing of social information in naturalistic environments.

\section{Method}

\subsection{Participants}

The study was preregistered with the Open Science Framework (https://osf.io/4kscq). To pre-select participants based on social anxiety traits, a pre-screening was programmed with SoSci survey (https://soscisurvey.de/). One-hundred healthy participants between 18 and 35 years completed the Social Interaction Anxiety Scale (SIAS) questionnaire (Stangier, Heidenreich, Berardi, Golbs, \& Hoyer, 1999) online. This 20-items questionnaire with a five-point response scale was first developed and validated by Mattick and Clarke (1998) and evaluated and translated into German by Stangier et al. (1999). The questionnaire assesses a general fear of social interactions, with higher scores indicating higher levels of social anxiety. Based on diagnostic criteria for social phobia from the diagnostic manual of mental disorders (DSM, American Psychiatric Association [APA], 2013), SIAS is widely used in research and clinical practice. Internal consistency was high for the current study $(\alpha=.92)$. In return for participation in the pre-screening, participants took part in a raffle for two amazon vouchers $(10 €)$. 
Based on the resulting distribution of SIAS scores, four quantiles were formed, with four equally sized sections based on the respective median $(M d n=18$, min $=2$, max $=50, Q 0.25=11.75, Q 0.5=18, Q 0.75=$ 29.25). Ten subjects were randomly selected from each quantile and invited via e-mail to participate in the subsequent EEG experiment in order to ensure a sufficient variance of SIAS scores in the EEG data $(M$ $=20.175, S D=11.703, \min =3, \max =49$ ). The sample size was based on previous studies (Hammerschmidt, Kulke, Broering, \& Schacht, 2018; Kulke, 2019; Kulke et al., 2016a). Eleven subjects did not reply and were replaced by other participants randomly selected from the respective quantiles. Three additional participants needed to be excluded and replaced due to excessive noise (over $50 \%$ lost trials) in the eye-tracking $(n=1)$ or ERP data $(n=2)$. The final sample contained fourteen male and twenty-six female participants (age range 19-29 years, $M=23.2, S D=2.7$ ). No participant needed to be excluded due to a score above the clinical cut-off value (32+) of the Autism Quotient Questionnaire (Baron-Cohen, Wheelwright, Skinner, Martin, \& Clubley, 2001). The study was approved by the local ethics committee and in line with the Declaration of Helsinki. All participants gave written informed consent prior to participation in the study.

\subsection{Stimuli and Materials}

Face Stimuli. The stimuli comprised 30 colorful faces from ten different individuals (5 male and 5 female), selected from the Karolinska Directed Emotional Faces database (Lundqvist, Flykt, \& Öhman, 1998), which has been validated, showing that participants correctly identify the displayed emotions (Goeleven, De Raedt, Leyman, \& Verschuere, 2008), each displaying angry, neutral, and happy facial expressions. Images were ellipsoid with a

\footnotetext{
${ }^{1}$ In order to ensure that emotions can be perceived at this eccentricity, a pilot study was conducted in which participants who were blind to the aim and had not previously seen the images correctly identified $95 \%$ of expressions at this
}

size of $4.5 \times 7 \mathrm{~cm}$ (corresponding to a visual angle of $3.4^{\circ} \times 5^{\circ}$ for the viewing distance of $80 \mathrm{~cm}$ ) and consisted of 324 x 504 pixels. Stimuli were identical to the ones used by (Kulke, 2019) and trimmed to exclude external features such as hair, ears and clothing and controlled for luminance, if required (Hammerschmidt et al., 2017) . All images were displayed on a white background on a liquid crystal display (LCD) computer screen with a $60 \mathrm{~Hz}$ refresh rate.

\subsection{Procedure}

Prior to the start of the experiment, participants provided a written informed consent. After the EEG electrodes were applied and the eye-tracker was calibrated and validated (see details below), the experimental session began and subjects were instructed on the Go/No-go task. The trial sequence was programmed with Python and PsychoPy, based on previous research (Kulke, 2019), with additional Nogo trials. The frequencies of Go and No-go trials were equal (.50/.50), as in previous similar studies using a Go/No-go paradigm (Jodo \& Kayama, 1992; Kulke et al., 2016a; Recio, Schacht, \& Sommer, 2009). At the start of each trial, a fixation cross with a size of $0.7 \mathrm{~cm}$ ( 50 pixels, $0.5^{\circ}$ ) was presented at the center of the screen for a randomized interval of $1500-2500 \mathrm{~ms}$. If participants fixated within an area of 40 pixels $(0.56 \mathrm{~cm}$, $0.4^{\circ}$ ) around the fixation cross for at least 150 samples (corresponding to $333.33 \mathrm{~ms}$ at a sampling rate of $500 \mathrm{~Hz}$ ) at the end or following this interval, the fixation cross gaze-contingently changed its color from black to either blue or orange, indicating a Go or No-go condition (color assignment counterbalanced across participants). Simultaneously, with the color change of the fixation cross, a face stimulus was presented $5.6 \mathrm{~cm}\left(400\right.$ pixels, $\left.4^{\circ}\right)$ to the right or the left side ${ }^{1}$. Participants were instructed

eccentricity when it was controlled with eye tracking that they kept fixating centrally. This suggests that emotions were successfully identified at this eccentricity, with comparable recognition rates as in the KDEF validation by Goelevan et al. 
to move their eyes towards peripheral face stimuli in Go trials and withhold an eyemovement in No-go trials. In Go trials, the face disappeared when participants fixated within 40 pixels $\left(0.56 \mathrm{~cm}, 0.4^{\circ}\right)$ of the image for at least 150 samples (= $333.33 \mathrm{~ms}$ ). In No-go trials, the face disappeared after $700 \mathrm{~ms}$, and the next trial started.

The experiment consisted of twelve blocks with 100 trials each, with short breaks inbetween, and lasted approximately one hour. Each expression was randomly presented 100 times per side and per condition. The order of all trials was randomized.

At the end of the EEG experiment, participants completed the "Reading Mind in the Eye" test (Baron-Cohen, Wheelwright, Hill, Raste, \& Plumb, 2001), the German version of the Behavioral Inhibition/Behavioral Avoidance Scale (BIS/BAS; (Strobel, Beauducel, Debener, \& Brocke, 2001)), the BarrattImpulsiveness Scale short version (BIS-15; (Meule, Vögele, \& Kübler, 2011)), and the Autism Quotient Questionnaire (AQ; (Baron-Cohen, Wheelwright, Skinner, et al., 2001)). Participants received course credit or monetary reward $(17 €)$ in return for participation.

\subsection{Eye-tracking data processing}

Eye-movements from both eyes were recorded continuously throughout the experiment with a desktop-mounted eyetracker (Eyelink 1000, SR Research, Ontario, Canada) at a sampling rate of $500 \mathrm{~Hz}$. A chin-rest was used to minimize head movements and to ensure an average viewing distance of $80 \mathrm{~cm}$. Prior to the start of the experiment each participant completed a standardized nine-point calibration and validation procedure. At the beginning of each presentation block, an additional one-point calibration was

(2008; hit rate ranging from $62.64 \%$ for neutral up to $92.65 \%$ for happy faces). Previous research furthermore demonstrated clear neural effects of completed to ensure a constantly high data quality. After completion of the experiment, the raw eye-tracking data was preprocessed in Matlab version R2017a based on previous research (Kulke, 2019). Gaze position data was averaged across both eyes. Horizontal saccades were determined as a gaze change in $\mathrm{x}$-position of more than $0.4^{\circ}$ between two subsequent samples (see e.g., Kulke, 2015, 2019; Kulke et al., 2016a). The latency of the first saccade after target onset was computed in Go-trials. Saccades occurring faster than $100 \mathrm{~ms}$ after stimulus onset were rejected as they are unlikely to be target-related and saccades slower than $700 \mathrm{~ms}$ were rejected as too slow. Furthermore, trials were excluded from further analysis if the fixation at the beginning of each trial was not within an area of $0.4^{\circ}$ around the fixation cross, if too many changes in fixation position were visible, indicative of noisy data or if eyemovements were incorrect $(M=8.5 \%$ of trials excluded). Errors (i.e. accidental eyemovements) were determined in No-go trials.

\subsection{EEG data processing}

EEG was recorded at a sampling rate of 512 $\mathrm{Hz}$ from 64 active $\mathrm{Ag}-\mathrm{AgCl}$ electrodes mounted in an elastic electrode cap (EasyCap, BioSemi, Amsterdam, the Netherlands) in line with the extended 1020 international system (Pivik et al., 1993). Additionally, six external electrodes were placed below the eyes (2), on the outer edges of the eyes (2) and to the left and right mastoids (2). Recordings were made with the common mode sense electrode (CMS) and the driven right leg passive electrode (DRL) as reference and ground electrodes. EEG was recorded using the ActiView707 BioSemi recording software for Linux. Electrode offsets were kept below $+/$ $25 \mathrm{mV}$.

Offline processing was conducted in Matlab version R2017a and the EEGlab toolbox

emotional expression when faces were presented at such an eccentricity (Kulke, 2019). 
(Delorme \& Makeig, 2004), based on previous research (Kulke, 2019; Kulke et al., 2016a). Trials with erroneous responses (errors of commission and omission) were excluded. External channels were removed, and continuous data was baseline-corrected using a $200 \mathrm{~ms}$ time interval prior to face stimulus onset. Filtering processes were done with a 2nd-order Butterworth bandpass filter with a high-pass boundary of $0.01 \mathrm{~Hz}$ and a low-pass boundary of $25 \mathrm{~Hz}$. Very low high-pass filters were used to avoid filter distortions in data with taskrelevant eye-movements (Kulke \& Kulke, 2020). To remove $50 \mathrm{~Hz}$ line noise, the CleanLine plugin (Mullen, 2012) was used; note that this was applied after the low-pass filter of $25 \mathrm{~Hz}$ was applied because some residual line noise may remain even after the low-pass filter was applied. The EEG data was re-referenced offline to the average reference and down-sampled from $512 \mathrm{~Hz}$ to $500 \mathrm{~Hz}$ after baseline correction. The system delay between the trigger signal and the visual presentation on the computer monitor was determined to be $24 \mathrm{~ms}$, using a light-sensitive diode and the triggers were shifted accordingly. Trials with noisy eyetracking data were excluded from further EEG analysis.

An Independent Component Analysis (ICA) was conducted on a separate dataset, on which stronger high-pass filters of $1 \mathrm{~Hz}$ and a low-pass boundary of $40 \mathrm{~Hz}$ were applied. After extracting epochs of -200 to $1000 \mathrm{~ms}$ around stimulus onset, the ICA was conducted using the EEGLAB plug-in ADJUST (Mognon, Jovicich, Bruzzone, \& Buiatti, 2011). Two trained coders independently marked independent components (ICs) that were unambiguously eye components (vertical eye-movements, horizontal eye-movements, and blinks). ICs unanimously identified by both coders were rejected from the final data set $(M=4.375$

\footnotetext{
${ }^{2}$ Note that we originally planned to extract a short time interval of $180 \mathrm{~ms}$ for saccades, but that due to the ICA and for comparability reasons we
}

per participant, $S D=1.372, \min =2, \max =$ 9).

The ICA weights from the separate ICA dataset were then applied to the original dataset. EEG data was epoched based on previous research (Kulke, 2019) to $-0.2-1 \mathrm{~s}$ around the target stimulus. ${ }^{2}$ Trials were rejected, when the maximum voltage was larger than $+/-100 \mu \mathrm{V}$, a slope larger than $50 \mu \mathrm{V}$ occurred or the deviation from the mean distribution exceeded 5. A repeated measures ANOVA comparing numbers of excluded trials between condition showed an effect of Go / No-go condition on excluded trials, $F(1,39)=31.09, p<.001$, with more excluded trials in the Go $(M=$ $12.5, S D=8.05)$ than in the No-go condition $(M=10.1, S D=6.39)$, but this did not interact with the screen side on which stimuli appeared, $F(1,39)=0.01, p=.903$ or the expression, $F(2,78)=0.112, p=.894$, and there were no main effects of side, $F(1$, $39)=0.743, p=.394$, expression, $F(2,78)$ $=0.274, p=.761$, interaction of side and expression, $F(2,78)=0.375, p=.689$, or three-way-interaction, $F(2,78)=1.293, p=$ .280. This suggests that Go trials were more often excluded due to noise than No-go trials, although the difference was small (on average 2 trials).

Areas of interest for ERP components were based on previous research. The P1 was quantified in two lateral parieto-occipital clusters (left: PO7, PO3, O1, right: PO8, PO4, O2) and its peak amplitude and latency was determined within $100-180 \mathrm{~ms}$ after target onset, based on comparable overt attention shift studies (Kulke et al., 2016a). In correct response trials, the mean EPN amplitude was extracted between 250 and $300 \mathrm{~ms}$ after stimulus onset in an occipito-parietal electrode cluster including electrodes O1, O2, P9, P10, PO7 and PO8 (Kulke, 2019); the mean LPC amplitude in a time window between 400 and $600 \mathrm{~ms}$ after stimulus onset in an occipito-parietal

decided to extract all components within the larger interval. 
electrode cluster including $\mathrm{Pz}, \mathrm{POz}, \mathrm{PO} 3$ and PO4 (Kulke, 2019); the mean N170 amplitudes were quantified in a time window between 130-200 ms after stimulus onset in a posterior electrode cluster (P7, P8, PO7, PO8, PO3, PO4, PO5, PO6) (Hinojosa et al., 2015). The mean No-goN2 was determined only for correct No-go trails between $200-350 \mathrm{~ms}$ in a frontocentral electrode cluster (Fz, F3, F4, Cz, C3, $\mathrm{C} 4, \mathrm{FCz}$ ), based on previous research (Hepsomali, Hadwin, Liversedge, Degno, \& Garner, 2019; Righi, Mecacci, \& Viggiano, 2009; Sehlmeyer et al., 2010). The frontal response was quantified between 100 and $180 \mathrm{~ms}$ in a frontal cluster (F3, FC5, FC3, FC1, C3, F4, FC2, FC4, FC6, C4).

\subsection{Statistical analysis}

The statistical analysis was conducted as pre-registered with the Open Science Framework (https://osf.io/4kscq) unless noted otherwise and performed using $\mathrm{R}$ (Team, 2013, version 3.4.4.). Repeatedmeasure ANOVAs were conducted using the ezANOVA function version 4.4-0 (Lawrence, 2016, version 4.4-0)to investigate effects of expression (happy, angry and neutral) and task (Go/No-go) on saccade latencies, error-rates, and ERP amplitudes (as well as latency in the case of the P1). To investigate effects of different personality traits (Supplement A), linear regression analysis using the $1 \mathrm{~m}$ function and linear mixed-effects regression models (LMMs) using the lme function (Bates, Mächler, Bolker, \& Walker, 2015) were conducted. Follow-up t-tests were performed using the t.test function. Assumptions for all statistical models (depending on the test: sphericity, homoscedasticity and normal distribution) were tested based on indications by Field, Miles, and Field (2002). Although the assumptions were not met for every analysis, statistical models used in this study were relatively robust against such violations. Assumptions on variable types and non-zero variance in predictor variables were mostly fulfilled. Nevertheless, p- values should be interpreted with caution. Since error-rates were measured as probabilities between 0 and 1, they do not fit these assumptions in the current sample due to a natural zero point and constrained data. Therefore, an additional, nonpreregistered binary logistic regression analysis was computed using the $\log R e g$ function to determine whether the likelihood of an incorrect answer is greater given higher social anxiety or BIS-15 scores. A generalized eta squared $\left(\eta^{2}\right)$ was computed as an effect size for statistical models using the ezANOVA command. A correlation coefficient was calculated as an estimate of effect size, using the rcontrast function in $\mathrm{R}$ programmed by Field and colleagues (2012) and Cohen's D was computed for post hoc t-tests, both based on interpretations by Cohen (1988). Note that we preregistered to test directional hypotheses one-tailed and non-directional hypotheses two-tailed. For simplification, two-tailed results are reported in the manuscript with a cut-off value of $p<.10$ for directional and $\mathrm{p}<.05$ for nondirectional hypotheses. In addition to the preregistered analyses, Bayes Factors (BF) were calculated with the respective commands $1 \mathrm{mBF}$ and ttestBF (Morey \& Rouder, 2015), to investigate in which direction and to what extent the probabilities for null hypothesis and alternative hypothesis differ. To investigate if the No-go-N2 amplitude could be correctly interpreted as an inhibition marker, differences between Go and No-go conditions were explored with a t-test using the t.test function.

\section{Results}

Means, SDs and 95\% confidence intervals for the observed effects are reported in Table 1.

\subsection{Eye-tracking}

The current study used eye-tracking to investigate the mechanisms of overt and covert emotion-driven attention. Since behaviorally overt and covert shifts of attention differ, with the former involving 
execution of a saccade and the latter involving inhibition of eye-movements, different measures were used to test the effect of emotion on No-go and Go conditions. To this end, when participants did make an overt shift of attention (Go condition), the latency of saccades was compared between emotional expressions. When participants inhibited a saccade instead (No-go condition), the error rate was compared across emotion conditions.

3.1.1. Saccade latencies. Overall saccade latencies in the Go condition (Figure 1) were significantly affected by expression, $F(2,78)=2.845, p=.064, B F=0.787, \eta^{2}=$ .001 (note the cut-off p-value of .10 due to pre-registered one-sided testing). Planned follow up t-tests showed no significant difference between the angry and neutral faces, $t(39)=-1.234, p=.225, B F=0.345$, $d=0.039$ and between angry and happy faces, $\mathrm{t}(39)=-1.181, p=.245, B F=.326, d$ $=0.045$. However, there was a significant difference in saccade latencies between the happy and neutral faces, $t(39)=-2.351, p=$ $.024, B F=1.957, d=0.085$.

3.1.2. Error rates. Overall, errors, i.e. accidental eye-movements in the No-go condition occurred in $1.59 \%$ of trials (SD = $2.23 \%)$. Error rates were unaffected by expression, $F(2,78)=0.797, p=.454, B F=$ $0.150, \eta^{2}=.003$. Planned follow up t-tests showed no significant difference of error rates between the happy and neutral condition, $t(39)=1.119, p=.270, B F=$ $0.305, d=0.129$, between the angry and neutral condition, $t(39)=0.536, p=.595$, $B F=0.195, d=0.052$, or between the angry and happy condition, $t(39)=-0.776, p=$ $.443, B F=0.226, d=0.083$.

Similar results were found with an exploratory logistic regression analysis predicting response (correct or incorrect) from expression category, $\chi 2(2)=2.331, p$ $=.312$ with $\mathrm{R}^{2}<.001$, which uses the binary data structure (i.e. codes for each trial whether the response was correct or incorrect) instead of averaging across data samples to compute proportions and is therefore less prone to model assumption violations.

\subsection{Neural effects (ERPs)}

The first aim of the study was to identify the neural differences between overt and covert attention shifts to faces with different expressions. In the following, all latencies are reported in ms and all amplitudes in $\mu \mathrm{V}$.

3.2.1. P1 latency. The P1 latency (Figure 1) was significantly affected by expression, $F(2,78)=3.320, p=.041, \eta^{2}=.006$ (note however, $\mathrm{BF}=0.380)$ and task, $F(1,39)=$ 7.819, $p=.008, \eta^{2}=.012, \mathrm{BF}=10.705$, with no significant interaction, $F(2,78)=$ $0.667, p=.516, \eta^{2}=.002, \mathrm{BF}=0.141$. Wave plots and topographical plots are displayed in the bottom panel of Figure 1. The planned follow up t-tests showed a significant difference between angry and neutral faces, $t(39)=-2.738, p=.009, B F=$ $4.355, d=.210$. However, neither the difference between happy and neutral, $t$ (39) $=-1.280, p=.208, B F=0.364 d=.109$, nor the difference between happy and angry, $t(39)=-1.224, p=.229, B F=0.341, d=$ .160 , reached statistical significance.

3.2.2. P1 amplitude. $P 1$ amplitude showed no overall effect of expression, $F(2,78)=$ 0.116, $p=0.890, \eta^{2}<.001, B F=0.047$, task, $F(1,39)=0.046, p=0.831, \eta^{2}<.001$, $B F=0.143$, or an interaction between task and expression, $F(2,78)=1.859, p=0.162$, $\eta^{2}<.001, B F=0.247$, on the P1 amplitude. Planned follow up t-tests showed neither significant differences between happy and neutral faces, $t(39)=0.507, p=.615, B F=$ $0.192, d=0.016$, nor between the neutral and angry faces, $t(39)=0.233, p=.817, B F$ $=0.175, d=0.007$, nor between the angry and happy faces, $t(39)=-0.238, p=.813$, $B F=0.175, d=.011$. 

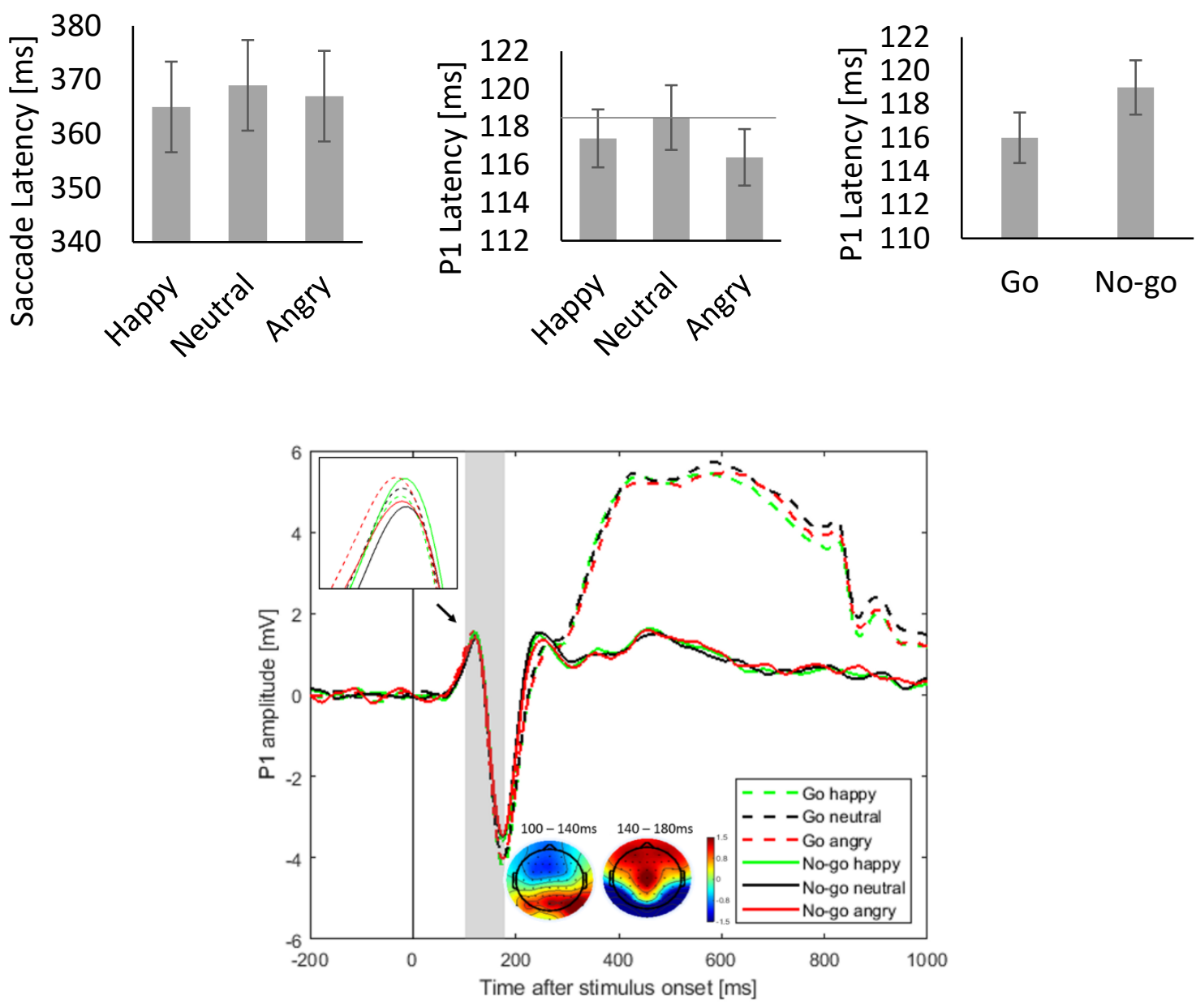

Figure 1. Upper panel: Mean latency of saccades (left panel) and P1 component (center panel), for happy, neutral and angry faces as well as mean P1 latency for Go and No-go conditions (right panel). Error bars indicate 2 standard errors around the mean (SE). The horizontal line signifies the neutral condition, to which the emotional expressions were compared. Bottom panel: Grand average wave plot, contrasted for happy, neutral, and angry faces in the Go (solid lines) and No-go (dashed lines) task. The embedded topographical plots depict the grand average scalp distribution between $100-140 \mathrm{~ms}$ and $140-180 \mathrm{~ms}$ after target onset across conditions. The grey bar represents the analysed time window (100-180ms).

3.2.3. Frontal response $100-180 \mathrm{~ms}$. ERP peak amplitudes in frontal regions (Figure 2) showed a significant effect of task, $F(1,39)=5.860, p=.020, \eta^{2}=.008, B F=$ 194.849, but no significant effect of expression, $F(2,78)=1.323, p=.272, \eta^{2}<$ $.001, B F=0.090$, and no significant interaction of task and expression, $F(1,78)$ $=0.604, p=.549, \eta^{2}<.001, B F=0.102$. Mean amplitudes were larger in the Go Table 1. compared to the No-go condition. The planned follow up t-tests showed no significant difference between the happy and the neutral, $t(39)=-1.693, p=.098, B F$ $=0.629 d=.066$, nor between the neutral and angry faces, $t(39)=-0.096, p=.924, B F$ $=0.171, d=.005$ or between the happy and angry faces, $t(39)=-1.357, p=0.183, B F$ $=0.398, d=.064$. 
Means, SDs and CIs of the observed effects.

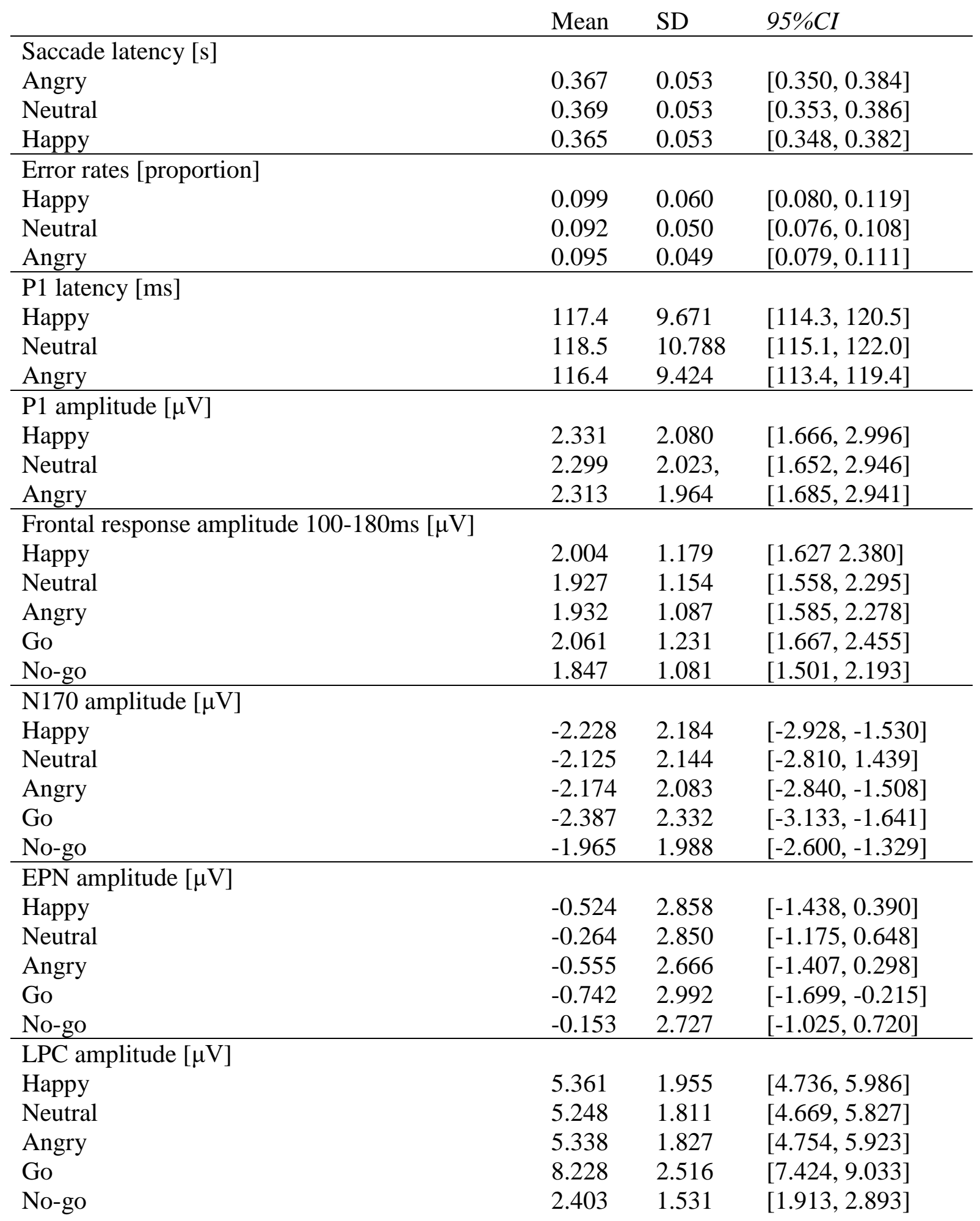



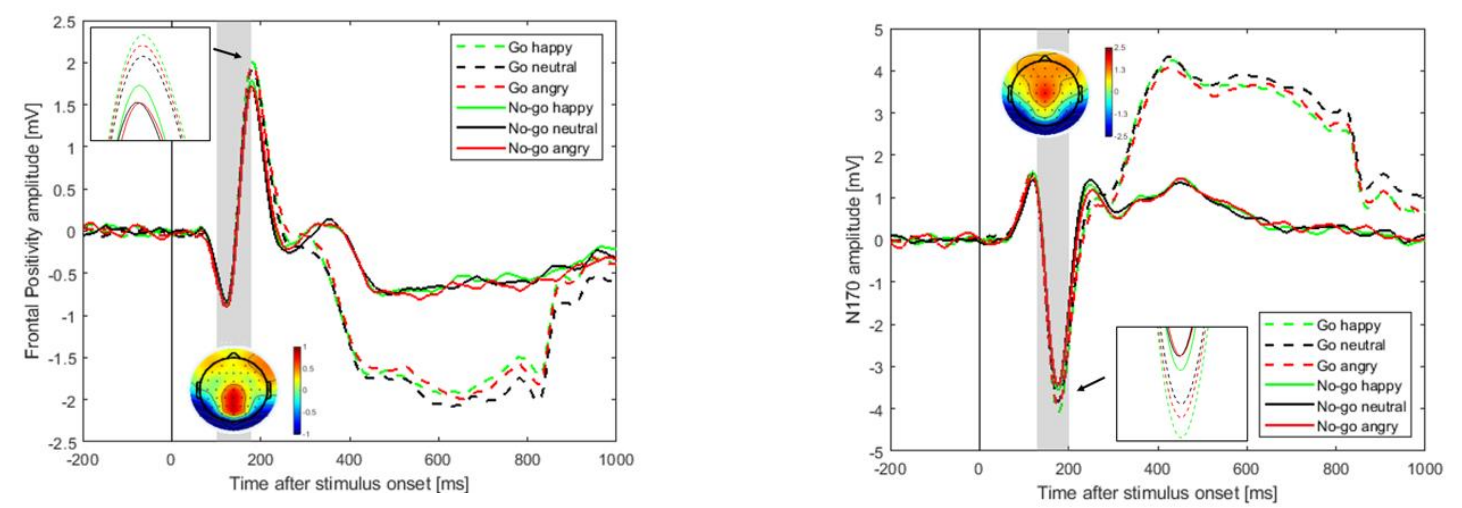

Figure 2. Topographical plots across all electrodes and wave of the electrode locations extracted to analyse the frontal positivity (left) and the N170 (right). The grey vertical bars represent the analysed time windows (100-180 ms after stimulus onset for the frontal response and 130-200 $\mathrm{ms}$ for the N170). The embedded topographical plots depict the grand average scalp distribution within the analysed time windows.

3.2.4. N170. The N170 amplitude (Figure 2, right panel) showed no significant effect of expression, $F(2,78)=1.152, p=.321, \eta^{2}<$ $.001, \quad B F=0.068$, nor a significant interaction between emotion category and condition, $\mathrm{F}(2,78)=0.466, \mathrm{p}=.630, \eta^{2}<$ $.001, B F=0.098$. However, there was a significant main effect of task, $F(1,39)=$ 9.439, $p=.004, \eta^{2}=.009, B F=46,841$. Planned follow up t-tests showed a significantly more negative amplitudes in the Go than the No-go trials, $t(39)=-3.072$, $p=.004, B F=9.270, d=0.180$. But there was no significant difference between the happy and neutral faces, $t(39)=-1.636, p$ $=.110, B F=0.578, d=0.048$, no difference between the neutral and angry faces, $t(39)=$ - 0.710, $p=.482, B F=0.216, d=0.023$, nor between the angry and happy ones, $t(39)$ $=0.756, p=.454, B F=0.223, d=0.026$.

3.2.5. EPN. EPN amplitude (Figure 3, left panel) was significantly affected by expression, $F(2,78)=6.796, p=.002, \eta^{2}=$ .002 (note however $\mathrm{BF}=0.311$ ) and by task, $F(1,39)=6.995, p=.012, \eta^{2}=.011$, $\mathrm{BF}=11021.88$, but there was no significant interaction, $F(2,78)=0.295, p=0.746, \eta^{2}$ $<.001, \mathrm{BF}=0.092$. Follow up planned $\mathrm{t}-$ tests revealed significantly more negative amplitudes in response to happy than to neutral faces, $t(39)=-3.100, p=.004, d=$ $0.091, B F=9.867$, as well as between to angry than to neutral faces, $t(39)=-3.316$, $p=.002, d=0.105, B F=16.662$. Differences between happy and angry faces were not significant, $t(39)=-0.349, p=$ $.729, d=0.011, B F=0.181$. Further, Go trials elicited larger negativities than No-go trials within the EPN time window, $t(39)=$ - 2.645, $p=.012, d=0.203, B F=3.562$.

3.2.6. LPC. LPC amplitude (Figure 3, right panel) was significantly affected by task, $F(1,39)=362.20, p<.001, \eta^{2}=.660, B F>$ $1,000,000$, with larger amplitudes in Go, than in No-go trials. There was no main effect of expression, $F(2,78)=1.192, p=$ $.309, \eta^{2}<.001, B F=0.045$, and no significant interaction, $F(2,78)=0.373, p=$ $.690, \eta^{2}<.001, B F=0.085$. Planned follow up t-tests showed significantly larger amplitudes in the Go than the No-go condition, $t(39)=19.032, p<.001, d=$ $2.555, B F>1,000,000$. There was no significant difference between the happy and neutral faces, $t(39)=1.321, p=.194, d$ $=0.060, B F=0.381$, nor between the angry and neutral ones, $t(39)=1.172, p=.250, d$ $=0.047, B F=0.322$ and the angry and happy faces, $t(39)=-0.329, p=.744, d=$ $0.012, B F=0.179$. 

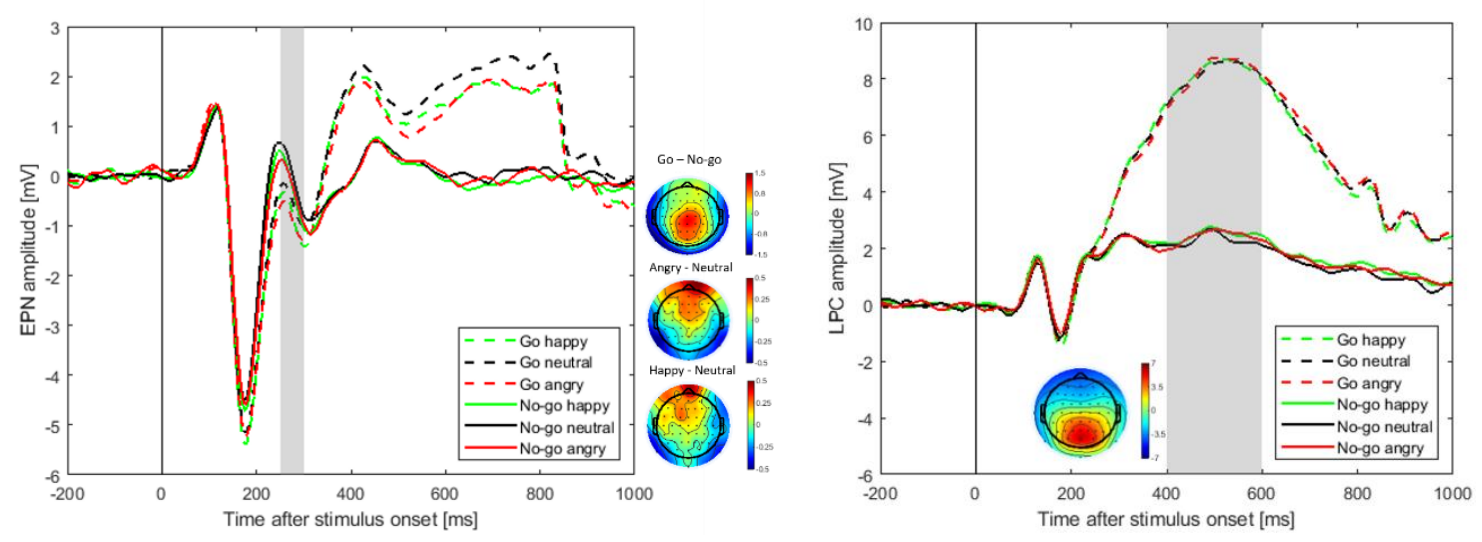

Figure 3. Topographical plots across all electrodes and waves of the electrode locations extracted to analyse the EPN (left panel) and LPC (right panel). The grey vertical bars represent the analysed time windows (250-300 ms after stimulus onset for EPN and 400-600 ms for the LPC). The topographical plots in the center display the difference between Go and No-go conditions, Angry and Neutral conditions and Happy and Neutral conditions within the EPN time window. The topographical plot in the right panel displays the grand average within the LPC time window across all conditions.

\section{Discussion}

The current study aimed to investigate the effect of emotional facial expression on the neural mechanisms of covert and overt shifts of attention to faces, as well as their relation to personality traits. In summary, the latency of saccades and of the P1 were significantly affected by the expression of the peripheral faces, although Bayesian statistics suggest that this effect is marginal. Saccades were faster towards positive (and marginally towards negative) than towards neutral faces. P1 responses were faster towards negative (and marginally towards positive) than neutral faces. In all other components - except for the EPN - only a main effect of task was found, with larger ERP amplitudes for the Go compared to the No-Go condition. On the EPN, we found a significant effect of emotional expression with larger responses to emotional than neutral faces and a significant effect of task. Interestingly, effects of facial expression and of condition (Go, i.e. overt shift vs. Nogo, i.e. covert shift) did not interact on any measures, confirmed by Bayes Factors that were considerably below the cut off criterion of $\mathrm{BR}<0.3$. This suggests that emotion-driven attention is comparable between overt and covert shifts.

\subsection{Differences in ERP responses between Go and No-go conditions}

We found that the P1 amplitude was comparable for covert and overt shifts, in line with previous overt attention shift research (Kulke et al., 2016a) indicating that early visual/perceptual processes are similar for the two types of attentional shift (Kulke, 2019; Kulke et al., 2016a). However, in later ERP components, several differences in neural responses between Go and No-go conditions were found. Across all later components examined here, larger response amplitudes were found in Go than in No-go conditions (as detected on the early frontal positivity, the N170, the EPN and the LPC). Only the N2 amplitude was larger in response to No-go compared to Go trials, as expected (Supplement A). A difference between covert and overt attention tasks is in line with previous research (Bokura et al., 2001; De Haan et al., 2008; Kulke et al., 2016a; A. C. Nobre, D. Gitelman, E. Dias, \& M.-M. Mesulam, 2000). The current study suggests that neural responses reflecting perceptual processing may be attenuated when an eyemovement needs to be inhibited, while only inhibitory responses are enhanced. This effect is unlikely to be related to eye- 
movement artefacts. Firstly, artefacts were removed using ICA. Secondly, effects were also found in posterior regions, which would only be marginally affected by eyemovements. Furthermore, effects were found both on positive ERPs (e.g. frontal positivity, LPC) as well as on negative components (N170). Due to the dipole structure underlying ERP measurements, it is possible that the frontal response reflects the other end of a dipole to occipital responses (for a discussion of this issue, see also Kulke et al., 2016a), as response amplitudes are larger, i.e. more negative for the N170 and more positive for the frontal response in Go than in No-go trials. However, interestingly, no such effects are found on the P1, which was measured in the same time window as the frontal response (100-180ms after target onset), while the time window of the N170 only partially overlaps with the frontal response window (130-200ms). Future research could further disentangle these observed scalp potentials through source analysis. Furthermore, the frontal positivity may be related to the $\mathrm{N} 2$, which was measured at a later time in the current study, but whose beginning may overlap with the peak of the frontal positivity according to the wave plots. However, the findings still suggest that overall response amplitudes are enhanced in Go- compared to No-go conditions. If eyemovement artefacts caused the effect, the polarity shift due to the eye-movement would only be directed either towards negative or positive directions, but not differentially effect different time windows. Finally, eye-movements induce a positive artefact on the ipsilateral and a negative one on the contralateral side. However, all electrode clusters were selected symmetrically, with equal numbers of electrodes in each hemisphere. Therefore, the observed effects most likely reflect distinct neural processes underlying covert and overt shifts of attention.

One possible explanation is that stimuli that one cannot look at are generally processed less intensively than those that can be viewed. This way, the brain may be favouring objects, from which it can derive more information through the execution of eye-movements, as is the case in the Gotask. Alternatively, the effort required to inhibit an eye-movement in No-go tasks may deplete processing resources, leaving a smaller processing capacity for neural responses to the stimuli. This would be in line with the "Premotor Theory of Attention", suggesting that attention automatically facilitates actions such as saccades towards a location (Eimer et al., 2005; Sheliga, Riggio, \& Rizzolatti, 1994) and these automatically generated motor responses need to be inhibited.

Another aspect to be considered is that the presentation of targets in No-go trials was constantly peripheral, while there was a change between peripheral and foveal presentation in Go trials due to the change in gaze position. Previous research suggests that emotion perception may differ between fovea and peripheral visual field (Rigoulot et al., 2011). In the current study, however, the average latency of eye-movement onsets was longer than $350 \mathrm{~ms}$ and all ERPs - except for the LPC - were computed in time windows before the saccades. Therefore, these ERPs should be unaffected by differences between peripheral and foveal stimulation. As ERP differences between Go and No-go conditions were not limited to the LPC and as no interaction occurred between condition and expression at LPC level, the observed effects are unlikely related to differences in foveal and peripheral stimulation.

In general, neural responses seem significantly larger in response to stimuli that participants can overtly shift to, compared to those they can only covertly attend to.

\subsection{Effects of emotional facial expression}

The EPN was significantly enhanced in response to happy and angry compared to neutral faces, in line with previous research with (Kulke, 2019) and without eyemovements (Schacht \& Sommer, 2009; 
Schupp et al., 2004). This confirms that participants perceived differences between emotional and neutral facial expressions in the current study.

Facial expression had a significant effect on P1 latency and saccade latency, with both latencies being shorter for emotional compared to neutral faces. However, this effect was marginal based on Bayesian analyses. It should be noted that effects of emotional expression were considerably smaller than effects of Go / No-go condition. These effects seem to be more variable and may require more power and larger sample sizes to be detected. They should therefore not be considered on an individual subject level but only on a group level. Interestingly, despite the latency effects in the current study, the amplitude of the P1 was unaffected by facial expression, with Bayesian statistics suggesting that a null effect is 21 times more likely than a significant effect. The lack of emotiondriven attention effects on the P1 amplitude in our study is in line with some covert attention studies (Frühholz et al., 2011; Rossignol et al., 2012), while contradicting others (Pourtois et al., 2005; Rellecke et al., 2012; Vlamings et al., 2009). These heterogenous findings are a recent topic of scientific discussion (Schindler \& Bublatzky, 2020), with the current study adding to this discussion by suggesting a lack of emotion effects on P1 amplitude in attention shift paradigms involving overt and covert shifts. The finding that emotional content can have small but significant effects on P1 latency but not on P1 amplitude suggests that emotional content might only effect the processing speed at early neural level, but not lead to a deeper processing as indicated by higher response amplitudes.

Previous research also found mixed results in regards to whether or not saccades are affected by emotional content (Bannerman et al., 2012; Kissler \& Keil, 2008; Kulke, 2019; Nummenmaa et al., 2006). In particular, the current results contrast the overt attention shift study by Kulke (2019), which showed that fast and reflexive eyemovements were unaffected by emotional content. In contrast to this previous study, in which eye-movements were purely reflexive, the current study involved a paradigm in which participants had to evaluate first whether they should make an eye-movement or not. Therefore, the eyemovements needed to be explicitly controlled and were less reflexive. Our results confirm the idea that explicit control was required due to increased task difficulty, as eye-movements were considerably slower (mean saccade latency between 365 and $369 \mathrm{~ms}$ ) than in the study by Kulke (2019) (188ms). Considering the differences in saccade latencies, while ERP latencies were comparable between both studies, more ERP components occurred before saccade onset in the present study. Therefore, more neural processing stages were completed before saccades were executed. Due to this delay, in the current study later neural responses could have impacted the eye-movements, while the fast reflexive eye-movements in the previous study (Kulke, 2019) could not have been affected by these later processing steps. It is therefore possible, that eye-movements are only affected by emotional facial expressions when certain neural processing of these expressions has occurred before saccade onset. In particular, the EPN occurred before saccade onset in the current study, while it was elicited after saccade onset in the study by Kulke (2019). This component also most reliably differentiated between facial expressions in both studies. Such a cortical differentiation, measured through the EPN, may affect saccade execution through feedback connections with the Superior Colliculus, which is highly linked with cortical areas, particularly the visual cortex. Taken together, the findings suggest that fast and reflexive saccades remain unaffected by emotional expressions; yet, if saccades are inhibited long enough for neural processing of emotional content to occur (i.e. until after the EPN), the subsequent eye-movements are influenced by these neural processes. 
Slower, controlled eye-movements can therefore be affected by emotional content, while fast, reflexive ones cannot.

\subsection{Methodological factors influencing saccade latency}

Several factors may have led to the overall slower eye-movements in the current study. In contrast to previous work (Kulke et al., 2016a), Go- and No-go-trials were randomized within and not between experimental blocks. Therefore, an additional processing effort was required in each trial to evaluate whether the task is to make an eye-movement or not. Furthermore, in comparison to studies using a comparable experimental design, target stimulus and cue (i.e. colour change) were presented simultaneously (Kissler \& Keil, 2008; Wieser, Pauli, \& Mühlberger, 2009). The evaluation of the cue may have occupied processing resources, leading to longer overall processing. Additionally, the colour change in the fixation center may additionally have attracted attention to the center thereby slowing the response to the periphery. Studies showed that competition between central and peripheral stimuli can decelerate both eye-movements and neural responses (Kulke et al., 2020). Therefore, the central competition for attention may have slowed responses in the current paradigm. The visual change in the fovea may also have induced simultaneous P1 responses, which may have overshadowed any emotion effects in the current study. To disentangle potential explanations for the deceleration of saccades, the use of a block design for Go and No-go trials in future research could minimize these distractions (see Kulke et al., 2016a). As the shift latency is decelerated in the current task, block designs and interleaved designs could furthermore be directly compared. In particular, it would be interesting to include a block in which participants are allowed to move their eyes freely, allowing an investigation of natural eye-movements. While the current study implemented a highly controlled comparison of covert and overt shifts of attention and instructed participants when to move their eyes (to ensure that sufficient numbers of trials with and without eye-movements were recorded), allowing both types of shifts closely simulates natural gaze conditions and thus provides one additional step in comparing controlled laboratory tasks and natural real-world eye-movements.

\subsection{Conclusion}

In summary, neural mechanisms significantly differed between covert and overt attention shifts in regards to various ERPs. Emotional facial expressions affected the EPN amplitude, the P1 latency and the saccade latency but did not interact with overt and covert conditions. Only slow saccades, such as measured in the current study, but not fast reflexive saccades, such as measured by Kulke (2019), seem to be affected by emotional expressions, with emotion effects being rather small, as neural processing of emotion may be required before saccade onset for emotion effects to occur. The current study suggests that neural responses are significantly enhanced when people move their eyes towards a face of interest, compared to when they need to inhibit such eye-movements. This finding indicates that the inhibition of gaze towards other people's faces, which is commonly observed during everyday life, has a significant impact on the magnitude of neural responses to these faces, although it does not seem to impair classification of their emotional expressions. Furthermore, our results suggest that emotional expressions automatically capture attention and thereby enhance neural responses regardless of whether shifts of attention are accompanied by eye-movements or not. The study is the first to implicate that effects of emotional expressions on early neural responses and saccades differ when people make reflexive eye-movements, compared to when they explicitly control and delay eye-movements towards faces. This highlights, once more, the relevance of studying overt attention under more realistic circumstances. In conclusion, the findings of our study indicate that the neuro- 
cognitive mechanisms of emotion-driven attention are independent of the overt or covert mode of the attention shift.

\section{Ethics statement}

The study abided by the ethics regulations of the Declaration of Helsinki and was approved by the local ethics committee of Georg-August University Göttingen. Written informed consent was obtained from participants before their participation in the study.

\section{Author contribution statement}

All authors conceptualised and designed the study. LB was involved in the data collection, supervised by LK. All authors conceived the data analysis, which was programmed and conducted by LB and LK. LK drafted the manuscript, which was critically revised by all authors.

\section{Data availability statement}

The data will be published as supplement alongside the article.

\section{Declaration of interest}

The authors declare no conflict of interest.

\section{Acknowledgements}

We would like to thank Nicolas Zdun for assistance during data collection. This project was supported by the LeibnizScienceCampus Primate Cognition. AP was supported by an ERC Starting Grant (716846).

\section{References}

American Psychiatric Association. (2013). Diagnostic and statistical manual of mental disorders (DSM-5®): American Psychiatric Pub.

Anllo-Vento, L., \& Hillyard, S. A. (1996). Selective attention to the color and direction of moving stimuli: electrophysiological correlates of hierarchical feature selection. Perception \& Psychophysics, 58(2), 191-206.

Argyle, M., \& Cook, M. (1976). Gaze and mutual gaze. Cambridge, Massachusetts: Cambridge University Press.

Atkinson, J., Hood, B., Braddick, O., \& Wattam-Bell, J. (1988). Infants control of fixation shifts with single and competing targets-mechanisms of shifting attention Perception, 17(3), 367-368.

Atkinson, J., Hood, B., Wattam-Bell, J., \& Braddick, O. (1992). Changes in infants' ability to switch visual attention in the first three months of life. Perception, 21, 643-643.

Bannerman, R. L., Hibbard, P. B., Chalmers, K., \& Sahraie, A. (2012). Saccadic latency is modulated by emotional content of spatially filtered face stimuli. Emotion, 12(6), 1384.

Baron-Cohen, S., Wheelwright, S., Hill, J., Raste, Y., \& Plumb, I. (2001). The "Reading the Mind in the Eyes" Test revised version: a study with normal adults, and adults with Asperger syndrome or high-functioning autism. The Journal of Child Psychology and Psychiatry and Allied Disciplines, 42(2), 241-251.

Baron-Cohen, S., Wheelwright, S., Skinner, R., Martin, J., \& Clubley, E. (2001). The autismspectrum quotient (AQ): Evidence from asperger syndrome/high-functioning autism, malesand females, scientists and mathematicians. Journal of Autism and Developmental Disorders, 31(1), 5-17.

Bates, D., Mächler, M., Bolker, B., \& Walker, S. (2015). Fitting Linear Mixed-Effects Models Using lme4. 2015, 67(1), 48. doi:10.18637/jss.v067.i01

Batty, M., \& Taylor, M. J. (2003). Early processing of the six basic facial emotional expressions. Cognitive brain research, 17(3), 613-620.

Bayle, D. J., Schoendorff, B., Hénaff, M.-A., \& Krolak-Salmon, P. (2011). Emotional facial expression detection in the peripheral visual field. PloS one, 6(6). 
Beauchamp, M. S., Petit, L., Ellmore, T. M., Ingeholm, J., \& Haxby, J. V. (2001). A parametric fMRI study of overt and covert shifts of visuospatial attention. Neuroimage, 14(2), 310321.

Belopolsky, A. V., \& Theeuwes, J. (2012). Updating the premotor theory: the allocation of attention is not always accompanied by saccade preparation. Journal of experimental psychology: human perception and performance, 38(4), 902.

Bentin, S., Allison, T., Puce, A., Perez, E., \& McCarthy, G. (1996). Electrophysiological studies of face perception in humans. Journal of cognitive neuroscience, 8(6), 551-565.

Bokura, H., Yamaguchi, S., \& Kobayashi, S. (2001). Electrophysiological correlates for response inhibition in a Go/NoGo task. Clinical neurophysiology, 112(12), 2224-2232.

Bradley, B. P., Mogg, K., \& Millar, N. H. (2000). Covert and overt orienting of attention to emotional faces in anxiety. Cognition and emotion, 14(6), 789-808. doi:10.1080/02699930050156636

Carver, C. S., \& White, T. L. (1994). Behavioral inhibition, behavioral activation, and affective responses to impending reward and punishment: the BIS/BAS scales. Journal of personality and social psychology, 67(2), 319.

Cohen. (1988). Statistical Power Analysis for the Behavioral Sciences: Jacob Routledge.

De Haan, B., Morgan, P. S., \& Rorden, C. (2008). Covert orienting of attention and overt eye movements activate identical brain regions. Brain research, 1204, 102-111.

Delorme, A., \& Makeig, S. (2004). EEGLAB: an open source toolbox for analysis of singletrial EEG dynamics including independent component analysis. Journal of neuroscience methods, 134(1), 9-21.

Dimigen, O., Sommer, W., Hohlfeld, A., Jacobs, A. M., \& Kliegl, R. (2011). Coregistration of eye movements and EEG in natural reading: analyses and review. Journal of Experimental Psychology: General, 140(4), 552.

Eimer, M. (1993). Effects of attention and stimulus probability on ERPs in a Go/Nogo task. Biological psychology, 35(2), 123-138.

Eimer, M. (2000). Event-related brain potentials distinguish processing stages involved in face perception and recognition. Clinical neurophysiology, 111(4), 694-705.

Eimer, M., Forster, B., Velzen, J. V., \& Prabhu, G. (2005). Covert manual response preparation triggers attentional shifts: ERP evidence for the premotor theory of attention. Neuropsychologia, 43(6), 957-966.

Eimer, M., \& Holmes, A. (2002). An ERP study on the time course of emotional face processing. Neuroreport, 13(4), 427-431.

Eimer, M., Van Velzen, J., \& Driver, J. (2002). Cross-modal interactions between audition, touch, and vision in endogenous spatial attention: ERP evidence on preparatory states and sensory modulations. Journal of cognitive neuroscience, 14(2), 254-271.

Eysenck, M. W., Derakshan, N., Santos, R., \& Calvo, M. G. (2007). Anxiety and cognitive performance: attentional control theory. Emotion, 7(2), 336.

Fairhall, S., Indovina, I., Driver, J., \& Macaluso, E. (2009). The brain network underlying serial visual search: comparing overt and covert spatial orienting, for activations and for effective connectivity. Cerebral cortex, 19(12), 2946-2958.

Foulsham, T., Walker, E., \& Kingstone, A. (2011). The where, what and when of gaze allocation in the lab and the natural environment. Vision research, 51(17), 1920-1931.

Frühholz, S., Jellinghaus, A., \& Herrmann, M. (2011). Time course of implicit processing and explicit processing of emotional faces and emotional words. Biological psychology, 87(2), 265-274.

Gobel, M. S., Kim, H. S., \& Richardson, D. C. (2015). The dual function of social gaze. Cognition, 136, 359-364.

Goeleven, E., De Raedt, R., Leyman, L., \& Verschuere, B. (2008). The Karolinska directed emotional faces: a validation study. Cognition and emotion, 22(6), 1094-1118. 
Hamann, S., \& Canli, T. (2004). Individual differences in emotion processing. Current opinion in neurobiology, 14(2), 233-238.

Hammerschmidt, W., Kulke, L., Broering, C., \& Schacht, A. (2018). Money or smiles: Independent ERP effects of associated monetary reward and happy faces. PloS one, 13(10), 0206142. Retrieved from http://dx.doi.org/10.1371/journal.pone.0206142

Hammerschmidt, W., Sennhenn-Reulen, H., \& Schacht, A. (2017). Associated motivational salience impacts early sensory processing of human faces. Neuroimage, 156, 466-474.

Hayward, D. A., Voorhies, W., Morris, J. L., Capozzi, F., \& Ristic, J. (2017). Staring reality in the face: A comparison of social attention across laboratory and real world measures suggests little common ground. Canadian Journal of Experimental Psychology/Revue canadienne de psychologie expérimentale, 71(3), 212.

Hepsomali, P., Hadwin, J. A., Liversedge, S. P., Degno, F., \& Garner, M. (2019). The impact of cognitive load on processing efficiency and performance effectiveness in anxiety: evidence from event-related potentials and pupillary responses. Experimental brain research, 237(4), 897-909.

Hinojosa, J. A., Méndez-Bértolo, C., \& Pozo, M. A. (2010). Looking at emotional words is not the same as reading emotional words: Behavioral and neural correlates. Psychophysiology, 47(4), 748-757.

Hinojosa, J. A., Mercado, F., \& Carretié, L. (2015). N170 sensitivity to facial expression: A meta-analysis. Neuroscience \& Biobehavioral Reviews, 55, 498-509.

Hood, B., \& Atkinson, J. (1993). Disengaging visual attention in the infant and adult. Infant Behavior and Development, 16(4), 405-422.

Hopfinger, J. B., \& Mangun, G. R. (1998). Reflexive attention modulates processing of visual stimuli in human extrastriate cortex. Psychological Science, 9(6), 441-447.

Huber-Huber, C., Ditye, T., Fernández, M. M., \& Ansorge, U. (2016). Using temporally aligned event-related potentials for the investigation of attention shifts prior to and during saccades. Neuropsychologia, 92, 129-141.

Hunt, A. R., Cooper, R. M., Hungr, C., \& Kingstone, A. (2007). The effect of emotional faces on eye movements and attention. Visual Cognition, 15(5), 513-531.

Itier, R. J., \& Taylor, M. J. (2004). N170 or N1? Spatiotemporal differences between object and face processing using ERPs. Cerebral cortex, 14(2), 132-142.

Jodo, E., \& Kayama, Y. (1992). Relation of a negative ERP component to response inhibition in a Go/No-go task. Electroencephalography and clinical Neurophysiology, 82(6), 477482.

Junghöfer, M., Sabatinelli, D., Bradley, M. M., Schupp, H. T., Elbert, T. R., \& Lang, P. J. (2006). Fleeting images: rapid affect discrimination in the visual cortex. Neuroreport, 17(2), 225-229. Retrieved from https://journals.1ww.com/neuroreport/Fulltext/2006/02060/Fleeting_images_rapid_af fect discrimination_in.23.aspx

Kaltwasser, L., Hildebrandt, A., Recio, G., Wilhelm, O., \& Sommer, W. (2014). Neurocognitive mechanisms of individual differences in face cognition: a replication and extension. Cognitive, Affective, \& Behavioral Neuroscience, 14(2), 861-878.

Kanwisher, N., McDermott, J., \& Chun, M. M. (1997). The fusiform face area: a module in human extrastriate cortex specialized for face perception. Journal of Neuroscience, 17(11), 4302-4311.

Kissler, J., \& Keil, A. (2008). Look-don't look! How emotional pictures affect pro-and antisaccades. Experimental brain research, 188(2), 215-222.

Kulke, L. (2015). Cortical mechanisms of visual attention in typically developing infants and adults. UCL (University College London),

Kulke, L. (2019). Neural mechanisms of overt attention shifts to emotional faces. Neuroscience, $418,59-68$. 
Kulke, L., Atkinson, J., \& Braddick, O. (2015). Automatic detection of attention shifts in infancy: eye tracking in the fixation shift paradigm. PloS one, 10(12).

Kulke, L., Atkinson, J., \& Braddick, O. (2016a). Neural differences between covert and overt attention studied using EEG with simultaneous remote eye tracking. Frontiers in human neuroscience, 10.

Kulke, L., Atkinson, J., \& Braddick, O. (2016b). Neural mechanisms of attention become more specialised during infancy: Insights from combined eye tracking and EEG. Developmental psychobiology, 59(2), 250-260.

Kulke, L., Atkinson, J., \& Braddick, O. (2020). Relation Between Event-Related Potential Latency and Saccade Latency in Overt Shifts of Attention. Perception, 49(4), 468-483.

Kulke, L., \& Kulke, V. (2020). Combining eye tracking with EEG: Effects of filter settings on EEG for trials containing task relevant eye-movements. bioRxiv.

Laidlaw, K. E., Foulsham, T., Kuhn, G., \& Kingstone, A. (2011). Potential social interactions are important to social attention. Proceedings of the National Academy of Sciences, 108(14), 5548-5553.

Lawrence, M. (2016). Easy Analysis and Visualization of Factorial Experiments. Retrieved from http://github.com/mike-lawrence/ez. http://github.com/mike-lawrence/ez

Lundqvist, D., Flykt, A., \& Öhman, A. (1998). The Karolinska directed emotional faces (KDEF). CD ROM from Department of Clinical Neuroscience, Psychology section, Karolinska Institutet, 91(630), 2.2.

Martinez, A., Anllo-Vento, L., Sereno, M. I., Frank, L. R., Buxton, R. B., Dubowitz, D., . . . Hillyard, S. A. (1999). Involvement of striate and extrastriate visual cortical areas in spatial attention. Nature neuroscience, 2(4), 364-369.

Mattick, R. P., \& Clarke, J. C. (1998). Development and validation of measures of social phobia scrutiny fear and social interaction anxiety. Behaviour research and therapy, 36(4), 455-470.

Meule, A., Vögele, C., \& Kübler, A. (2011). Psychometrische evaluation der deutschen Barratt impulsiveness scale-Kurzversion (BIS-15). Diagnostica.

Mogg, K., \& Bradley, B. P. (2002). Selective orienting of attention to masked threat faces in social anxiety. Behaviour research and therapy, 40(12), 1403-1414.

Mogg, K., Philippot, P., \& Bradley, B. P. (2004). Selective attention to angry faces in clinical social phobia. Journal of abnormal psychology, 113(1), 160.

Mognon, A., Jovicich, J., Bruzzone, L., \& Buiatti, M. (2011). ADJUST: An automatic EEG artifact detector based on the joint use of spatial and temporal features. Psychophysiology, 48(2), 229-240.

Morey, R. D., \& Rouder, J. N. (Producer). (2015). BayesFactor: Computation of Bayes Factors for Common Designs. $R$ package version 0.9.12-2. Retrieved from http://CRAN.Rproject.org/package $=$ BayesFactor

Mulckhuyse, M., Talsma, D., \& Theeuwes, J. (2007). Grabbing attention without knowing: Automatic capture of attention by subliminal spatial cues. Visual Cognition, 15(7), 779788.

Mullen, T. (2012). CleanLine EEGLAB plugin. San Diego, CA: Neuroimaging Informatics Toolsand Resources Clearinghouse (NITRC).

Nasiopoulos, E., Risko, E. F., \& Kingstone, A. (2015). Social attention, social presence, and the dual function of gaze. In The Many Faces of Social Attention (pp. 129-155): Springer.

Nieuwenhuis, S., Yeung, N., \& Cohen, J. D. (2004). Stimulus modality, perceptual overlap, and the go/no-go N2. Psychophysiology, 41(1), 157-160.

Nobre, A., Gitelman, D., Dias, E., \& Mesulam, M. (2000). Covert visual spatial orienting and saccades: overlapping neural systems. Neuroimage, 11(3), 210-216. 
Nobre, A. C., Gitelman, D., Dias, E., \& Mesulam, M.-M. (2000). Covert visual spatial orienting and saccades: overlapping neural systems. Neuroimage, 11(3), 210-216.

Nummenmaa, L., Hyönä, J., \& Calvo, M. G. (2006). Eye movement assessment of selective attentional capture by emotional pictures. Emotion, 6(2), 257.

Peschard, V., Philippot, P., Joassin, F., \& Rossignol, M. (2013). The impact of the stimulus features and task instructions on facial processing in social anxiety: an ERP investigation. Biological psychology, 93(1), 88-96.

Phillips, M. L., Williams, L. M., Heining, M., Herba, C. M., Russell, T., Andrew, C., . . . Morgan, M. (2004). Differential neural responses to overt and covert presentations of facial expressions of fear and disgust. Neuroimage, 21(4), 1484-1496.

Pivik, R. T., Broughton, R. J., Coppola, R., Davidson, R. J., Fox, N., \& Nuwer, M. R. (1993). Guidelines for the recording and quantitative analysis of electroencephalographic activity in research contexts. Psychophysiology, 30(6), 547-558.

Pourtois, G., Dan, E. S., Grandjean, D., Sander, D., \& Vuilleumier, P. (2005). Enhanced extrastriate visual response to bandpass spatial frequency filtered fearful faces: Time course and topographic evoked-potentials mapping. Human Brain Mapping, 26(1), 65 79.

Pourtois, G., Grandjean, D., Sander, D., \& Vuilleumier, P. (2004). Electrophysiological correlates of rapid spatial orienting towards fearful faces. Cerebral cortex, 14(6), 619633.

Praamstra, P., \& Oostenveld, R. (2003). Attention and movement-related motor cortex activation: a high-density EEG study of spatial stimulus-response compatibility. Cognitive brain research, 16(3), 309-322.

Rachman, S. (1980). Emotional processing. Behaviour research and therapy, 18(1), 51-60.

Recio, G., Schacht, A., \& Sommer, W. (2009). Effects of inter-stimulus interval on skin conductance responses and event-related potentials in a Go/NoGo task. Biological psychology, 80(2), 246-250.

Recio, G., Wilhelm, O., Sommer, W., \& Hildebrandt, A. (2017). Are event-related potentials to dynamic facial expressions of emotion related to individual differences in the accuracy of processing facial expressions and identity? Cognitive, Affective, \& Behavioral Neuroscience, 17(2), 364-380.

Rellecke, J., Palazova, M., Sommer, W., \& Schacht, A. (2011). On the automaticity of emotion processing in words and faces: event-related brain potentials evidence from a superficial task. Brain and cognition, 77(1), 23-32.

Rellecke, J., Sommer, W., \& Schacht, A. (2012). Does processing of emotional facial expressions depend on intention? Time-resolved evidence from event-related brain potentials. Biological psychology, 90(1), 23-32.

Rellecke, J., Sommer, W., \& Schacht, A. (2013). Emotion effects on the N170: a question of reference? Brain topography, 26(1), 62-71.

Righi, S., Mecacci, L., \& Viggiano, M. P. (2009). Anxiety, cognitive self-evaluation and performance: ERP correlates. Journal of Anxiety Disorders, 23(8), 1132-1138.

Rigoulot, S., D’Hondt, F., Defoort-Dhellemmes, S., Despretz, P., Honoré, J., \& Sequeira, H. (2011). Fearful faces impact in peripheral vision: behavioral and neural evidence. Neuropsychologia, 49(7), 2013-2021.

Risko, E. F., Richardson, D. C., \& Kingstone, A. (2016). Breaking the fourth wall of cognitive science: Real-world social attention and the dual function of gaze. Current Directions in Psychological Science, 25(1), 70-74.

Rossignol, M., Philippot, P., Bissot, C., Rigoulot, S., \& Campanella, S. (2012). Electrophysiological correlates of enhanced perceptual processes and attentional capture by emotional faces in social anxiety. Brain research, 1460, 50-62. 
Sabatini, E., Della Penna, S., Franciotti, R., Ferretti, A., Zoccolotti, P., Rossini, P. M., . . . Gainotti, G. (2009). Brain structures activated by overt and covert emotional visual stimuli. Brain research bulletin, 79(5), 258-264.

Schacht, A., \& Sommer, W. (2009). Emotions in word and face processing: early and late cortical responses. Brain and cognition, 69(3), 538-550.

Schindler, S., \& Bublatzky, F. (2020). Attention and emotion: An integrative review of emotional face processing as a function of attention. Cortex.

Schupp, H. T., Junghöfer, M., Weike, A. I., \& Hamm, A. O. (2004). The selective processing of briefly presented affective pictures: an ERP analysis. Psychophysiology, 41(3), 441449.

Sehlmeyer, C., Konrad, C., Zwitserlood, P., Arolt, V., Falkenstein, M., \& Beste, C. (2010). ERP indices for response inhibition are related to anxiety-related personality traits. Neuropsychologia, 48(9), 2488-2495.

Sheliga, B. M., Riggio, L., \& Rizzolatti, G. (1994). Orienting of attention and eye movements. Experimental brain research, 98(3), 507-522.

Shomstein, S., Kravitz, D. J., \& Behrmann, M. (2012). Attentional control: Temporal relationships within the fronto-parietal network. Neuropsychologia, 50(6), 1202-1210.

Stangier, U., Heidenreich, T., Berardi, A., Golbs, U., \& Hoyer, J. (1999). Die Erfassung sozialer Phobie durch Social Interaction Anxiety Scale (SIAS) und die Social Phobia Scale (SPS). Zeitschrift für klinische Psychologie.

Strobel, A., Beauducel, A., Debener, S., \& Brocke, B. (2001). Eine deutschsprachige Version des BIS/BAS-Fragebogens von Carver und White. Zeitschrift für Differentielle und diagnostische Psychologie.

Van der Stigchel, S., \& Theeuwes, J. (2007). The relationship between covert and overt attention in endogenous cuing. Perception \& Psychophysics, 69(5), 719-731.

Van Veen, V., \& Carter, C. S. (2002). The anterior cingulate as a conflict monitor: fMRI and ERP studies. Physiology \& behavior, 77(4-5), 477-482.

Vetter, P., Badde, S., Phelps, E. A., \& Carrasco, M. (2019). Emotional faces guide the eyes in the absence of awareness. ELife, 8, e43467.

Vlamings, P. H., Goffaux, V., \& Kemner, C. (2009). Is the early modulation of brain activity by fearful facial expressions primarily mediated by coarse low spatial frequency information? Journal of vision, 9(5), 12-12.

Weaver, M. D., van Zoest, W., \& Hickey, C. (2017). A temporal dependency account of attentional inhibition in oculomotor control. Neuroimage, 147, 880-894.

Wieser, M. J., Hambach, A., \& Weymar, M. (2018). Neurophysiological correlates of attentional bias for emotional faces in socially anxious individuals-Evidence from a visual search task and N2pc. Biological psychology, 132, 192-201.

Wieser, M. J., Pauli, P., \& Mühlberger, A. (2009). Probing the attentional control theory in social anxiety: An emotional saccade task. Cognitive, Affective, \& Behavioral Neuroscience, 9(3), 314-322.

Wieser, M. J., Pauli, P., Weyers, P., Alpers, G. W., \& Mühlberger, A. (2009). Fear of negative evaluation and the hypervigilance-avoidance hypothesis: an eye-tracking study. Journal of Neural Transmission, 116(6), 717-723.

Yamaguchi, S., Tsuchiya, H., \& Kobayashi, S. (1994). Electroencephalographic activity associated with shifts of visuospatial attention. Brain, 117(3), 553-562.

Yamaguchi, S., Tsuchiya, H., \& Kobayashi, S. (1995). Electrophysiologic correlates of visuospatial attention shift. Electroencephalography and clinical Neurophysiology, 94(6), 450-461.

Yang, L., \& Li, X.-B. (2014). Anxiety alters brain activity of response inhibition: Evidence from event-related potentials and source current density analysis. Paper presented at the 2014 4th IEEE International Conference on Information Science and Technology. 


\section{Supplement A: Individual Differences}

Emotional processing differs between individuals (Hamann \& Canli, 2004; Kaltwasser et al., 2014; Recio et al., 2017) and these differences should not be ignored, as they may profoundly change the interpretations of experimental findings. Therefore, a second aim of the current study was to explore personality-related differences in the expected effects of attention and emotion. Different personality traits have been suggested to be related to emotional face perception (Rachman, 1980). In particular, social anxiety and its relation to emotional attention constitutes an area of interest to society. According to the attentional control theory (Eysenck, Derakshan, Santos, \& Calvo, 2007), anxiety facilitates the detection of threat and therefore, anxious individuals involuntary allocate initial attentional resources to salient threat-related stimuli (Mogg et al., 2004). People with high levels of social anxiety preferred to look at threatening compared to neutral faces (Bradley et al., 2000), detected angry faces more easily in a visual search paradigm (Wieser et al., 2018), more often looked first towards emotional than towards neutral faces (Wieser, Pauli, Weyers, et al., 2009), and showed an attention bias towards faces and threat-related facial expressions due to their increased fear of negative social evaluation (Mogg \& Bradley, 2002). They also show a hypervigilance to faces in general, i.e., independent of their facial expressions (Peschard, Philippot, Joassin, \& Rossignol, 2013; Rossignol et al., 2012). The general bias towards faces and emotional expressions is also reflected in difficulties to inhibit responses towards them (cf. Eysenck et al., 2007). In an antisaccade task towards faces with different expressions, socially anxious individuals made more erratic saccades than controls (Wieser, Pauli, \& Mühlberger, 2009). To investigate the special role of social anxiety during attention shifts to faces, the current study recruited participants with a wide range of anxiety traits, based on a pre-screening. We expected participants with higher anxiety scores to shift faster to angry compared to neutral faces and to make more errors in the No-go condition, particularly in response to angry faces. On a neural level, such a response inhibition has been related to the No-go-N2, a fronto-central ERP between 200-350ms after stimulus onset that is larger in No-go than in Go trials (Eimer, 1993; Jodo \& Kayama, 1992). The N2 amplitude is larger the more effort is required to inhibit a response (Jodo \& Kayama, 1992; Nieuwenhuis, Yeung, \& Cohen, 2004; Van Veen \& Carter, 2002). Higher No-go-N2 amplitudes were found for anxious in comparison to non-anxious individuals (Righi et al., 2009; Sehlmeyer et al., 2010), indicating enhanced cognitive control required to inhibit prepotent responses (Righi et al., 2009). In contrast, Yang and Li (2014) and Hepsomali et al. (2019) found no difference in No-go-N2 depending on trait anxiety. We expected larger ERP amplitudes for participants with higher trait social anxiety of the P1 and No-go-N2, depending on emotion category. In summary, due to the societal relevance and the particular abundance on clinical and non-clinical research on effects of anxiety on attention shifts, the current study was specifically tailored to measuring a wide spectrum of social anxiety traits by pre-selecting participants based on a pre-screening of social anxiety.

Additionally, further individual differences may also play a role during attention shifts to emotional faces. Some people show impairments in processing of social stimuli such as faces, for example people with autism (American Psychiatric Association, 2013). The better people are at recognizing emotional expressions from faces (measured through the Reading Mind in the Eye Test (Baron-Cohen, Wheelwright, Hill, et al., 2001)), the larger we expect any observed emotion effects to be. Motivational tendencies for approach towards reward or avoidance of punishment (BAS/BIS) can affect low-level behavior (Carver \& White, 1994). We expected people who are more prone to avoidance of punishment to shift faster to angry faces, as they would draw their attention more quickly, and people who show greater reward-seeking behavior to shift faster to happy faces. Impulsiveness can affect reflexive eye-movement behavior, leading to an inability to inhibit eye-movements towards appearing stimuli. We therefore expected participants with higher impulsiveness to make more errors in the No-go condition. 


\section{Results}

An overview of all questionnaire scores can be found in Supplement D. Results of the preregistered tests of individual differences are displayed in Table S1. Models included random intercepts unless only between-participants effects were included. Of the preregistered measures, there was only a significant effect of Reading mind in the eye scores on saccade latency, $F(1,38)=8.20, p=.007, \eta^{2}=.175, \mathrm{BF}=1.168$, and an interaction effect of Reading mind in the eye scores and expression on P1 latency, $F(2,76)=4.023, p=.022, \eta^{2}=.009, B F$ $=1.595$, showing that $\mathrm{P} 1$ latency decreased with increasing RME scores in all conditions, but decreased less strongly in response to happy than to neutral faces.

As we only planned to investigate differences in the No-go $\mathrm{N} 2$ in relation to individual differences, we conducted an additional exploratory analysis to compare $\mathrm{N} 2$ amplitude between Go and No-go conditions using a dependent samples t-test, which confirmed that amplitudes were significantly more negative in the No-go condition than in the Go condition, $t(39)=3.165$, $p=.003, d=0.252, B F=11.547$. No-go N2 amplitude is displayed in Figure S1. Additional non-preregistered, exploratory logistic regressions showed effects of SIAS score and of BIS-15 score on error rates.

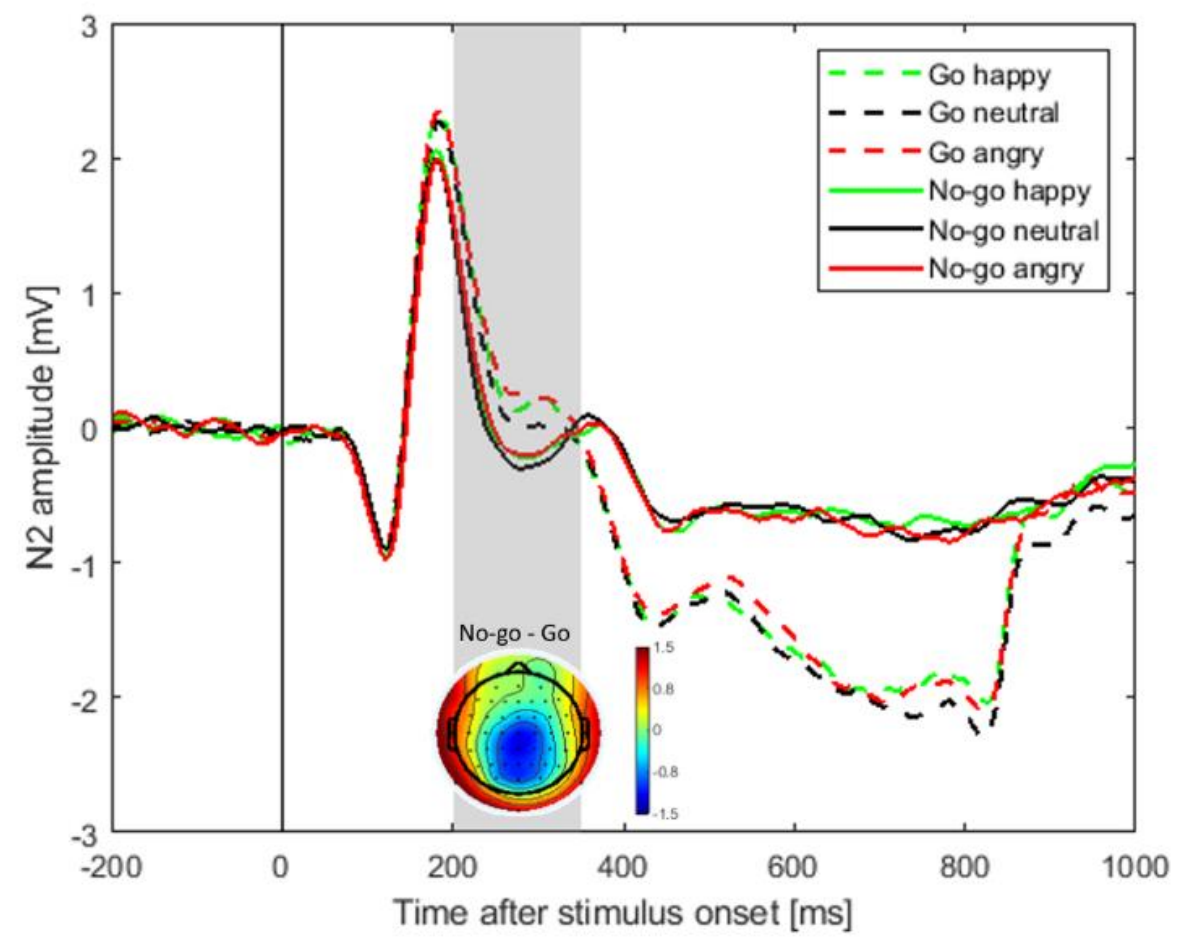

Figure S1. Wave and topographical plots of the No-go N2. 
Table 1.

Effects of personality traits

Personality Outcome Effects inventory measure

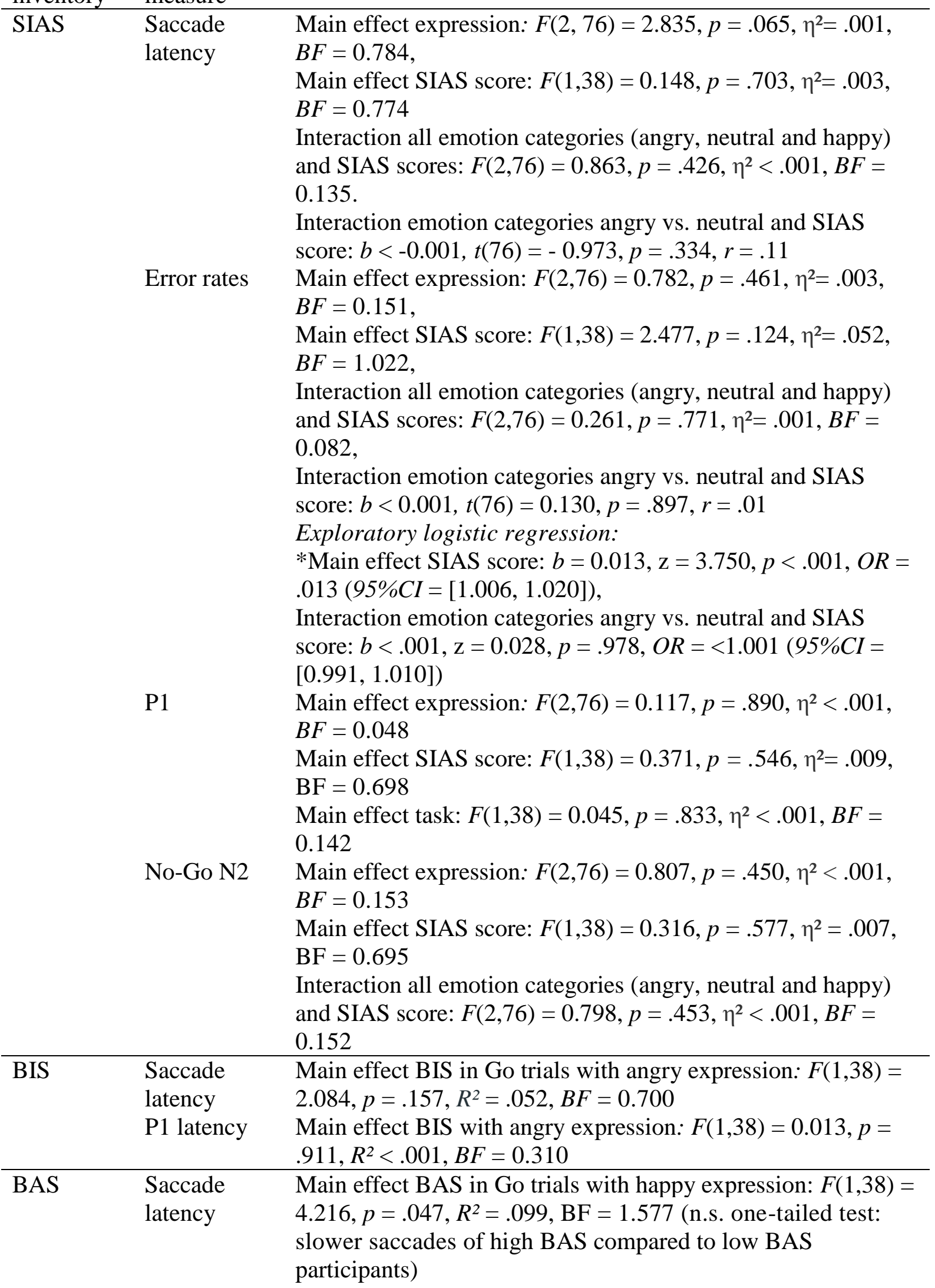




\begin{tabular}{|c|c|c|}
\hline & P1 latency & $\begin{array}{l}\text { Main effect BAS for happy expression: } F(1,38)=0.010, p= \\
.922, R^{2}<.001, \mathrm{BF}=0.310\end{array}$ \\
\hline BIS-15 & Error rates & $\begin{array}{l}\text { Main effect BIS-15: } F(1,38)=1.303, p=.261, R^{2}=.033, \mathrm{BF}= \\
0.517 \text {, } \\
\text { Exploratory logistic regression: } \\
\text { * Main effect BIS-15: } b=0.018, \mathrm{z}=4.285, p<.001 \text { with an } \\
\text { odds ratio of } 1.018(95 \% C I=[1.010,1.027])\end{array}$ \\
\hline \multirow[t]{2}{*}{$\begin{array}{l}\text { Reading } \\
\text { mind in } \\
\text { the eye }\end{array}$} & $\begin{array}{l}\text { Saccade } \\
\text { latency }\end{array}$ & $\begin{array}{l}* \text { Main effect of Reading mind in the eye scores, } F(1,38)= \\
8.20, p=.007, \eta^{2}=.175, \mathrm{BF}=1.168 ; \\
\text { Interaction of Reading mind in the eye score and expression, } \\
F(2,76)=0.609, p=.546, \eta^{2}<.001, \mathrm{BF}=0.105 \\
\text { Main effect Reading mind in the eye score: } F(1,38)=3.315, p \\
=.077, \eta^{2}=.074, \mathrm{BF}=1.357, \\
\text { *Interaction effect of Reading mind in the eye scores and } \\
\text { expression: } F(2,76)=4.023, p=.022, \eta^{2}=.009, B F=1.595 \text { : } \\
\text { P1 latency decreased with increasing RME scores in all } \\
\text { conditions, but decreased less strongly in response to happy } \\
\text { than to neutral faces }\end{array}$ \\
\hline & $\begin{array}{l}\text { EPN } \\
\text { amplitude }\end{array}$ & $\begin{array}{l}\text { Main effect Reading Mind in the eye scores: } F(1,38)=0.198 \\
p=.659, \eta^{2}=.005, B F=0.781 \text {, } \\
\text { Interaction of expression and Reading mind in the eye score: } \\
F(2,76)=0.433, p=.650, \eta^{2}<.001, B F=0.099\end{array}$ \\
\hline
\end{tabular}

\section{Discussion}

Contrary to our expectation based on previous theories and research (Eysenck et al., 2007; Rachman, 1980), differences in personality traits only had negligible effects on emotion-driven attention in the current study. It is possible that complex individual traits do not play a significant role during very basic functions such as the attention shifts measured in this study. The Bayesian statistics underline that personality effects are unlikely. However, the sample size in the current study was preregistered based on previous research. Other studies on individual differences in social anxiety tested smaller or comparable sample sizes (e.g. Mogg et al., 2004: 30 participants, Wieser et al., 2018: 42 participants, Wieser et al., 2009: 36 participants, Peschard et al., 2013: 36 participants, Rossignol at al., 2013: 26 participants), so that we oriented on these numbers. It is possible that previous research overestimated personality effects and that these effects are small, if they exists, so that larger samples need to be tested to reveal them (e.g. in a multi-lab approach). Indeed, post hoc power computations in our study show that the power to detect individual differences varies greatly (e.g. between $1-\beta=.05$ and $1-\beta>$.1). All individual differences should therefore be interpreted with caution. An increase in power was achieved by using logistic regressions to investigate personality traits. With this (non-preregistered) method, it was found that more impulsive people made more errors in the No-go condition. Furthermore, individuals who were more anxious also made more erratic saccades. This general deficit in saccade inhibition is in line with previous research by (Eysenck et al., 2007; Wieser, Pauli, \& Mühlberger, 2009), indicating that anxious individuals may suffer from an impairment of top-down attentional control and increased bottom up attention, resulting in a dysfunctional ability to inhibit a prepotent response. This impulse control was, however, not affected by emotional expression, contradicting the hypothesis that anxious individuals preferentially process threat (Bradley et al., 2000) and emotional faces (Wieser et al., 2018; Wieser, Pauli, Weyers, et al., 2009). Furthermore, no effects were found on a neural level. As the behavior in the current study results from several neural response processes, only the sum of these processes may reveal effects, while no single component on their own can best describe 
the modulation effects of social anxiety. It should further be noted that a non-clinical sample of participants was tested in the current study. Therefore, effects may only be apparent in more extreme populations, such as social anxiety patients.

Scores on the Reading Mind in the Eyes test had significant effects on saccade latency, indicating that the ability to identify emotions from faces may effect very early eye-movements towards them. There was an interaction effect of RME score and emotion category on P1 latency. The Reading Mind in the Eyes test measures participants' ability to identify emotions from faces (Baron-Cohen, Wheelwright, Hill, et al., 2001). Hence, the ability to identify emotions affects early responses to faces differing in emotional content.

Overall, personality effects may either be negligible or their effects could be too complex to reveal themselves in the preregistered tests in the current study. 